\title{
INVESTIGATION AND COMPARISON OF OPTICAL Properties of NiCKEL NANOWIRES AND NiCKEL ThIN FILM USING HE-NE LASER
}

\author{
Saleem Khan ${ }^{1}$, Sandeep Arya ${ }^{1}$, Manpreet Kaur ${ }^{2}$, Ashok Kumar ${ }^{2}$, Parveen Lehana ${ }^{1 *}$ \\ ${ }^{1}$ Dept. Of Physics \& Electronics, University of Jammu, Jammu-India \\ ${ }^{2}$ Ambala College of Engineering, Kurukshetra University, Haryana-India \\ *pklehanajournals@gmail.com
}

\begin{abstract}
Ni nanowires are synthesized by template based electrochemical technique. A commercially available filter membrane of alumina anodic oxide $(A A O)$ is used for the fabrication of nanowires. AAO membrane supported on copper substrate yields better hexagonal nanowires. Morphological studies of nanowires are carried out using scanning electron microscopy (SEM). The analysis shows the fabrication of vertical $100 \mathrm{~nm}$ nanowires. The optical properties using He-Ne laser light have been studied. In order to study the reflection of laser light from the substrate containing Ni nanowires a high resolution camera was employed. These images were further processed using image processing technique. To nullify the effect of the surrounding optical sources, these images were desaturated. The analysis of the results shows that at different angles the reflection of light from substrate containing Ni nanowires shows a regular pattern as compared to Ni thin film. Peak is observed when laser is incident at right angle to the plane of the sample containing Ni nanowires.
\end{abstract}

\section{KEYWORDS}

AAO membrane, electrodeposition, nanowires, wavelength, light intensity.

\section{INTRODUCTION}

Nanotechnology is based on the study of the control of matter on an atomic and molecular scale. It has the potential to create new materials and devices with applications in medicine, electronics, energy production, and also construction. Typically, an atom has a diameter of a few Angstroms $\left(1 \AA=0.1 \mathrm{~nm}=10^{-10} \mathrm{~m}\right)$, a molecule's size is a few $\mathrm{nm}$, and clusters or nanoparticles formed by hundreds or thousands of atoms have sizes of tens of nm [1-3]. There are two main approaches to nano-scale manufacturing. These have been labelled top-down and bottom-up by the nanotechnology community [4]. Top-down manufacturing methods consist of conventional micro-fabrication techniques including nano-lithography and chemical etching. A common characteristic of top-down technologies is the removal of material from bulk material to create the final product. Bottom-up manufacturing is an assembly process. Its goal is to create larger and more complex structures out of smaller basic components [5-7]. Studies of high aspect ratio structures with widths/thicknesses in the nanometer range, nanowires have revealed very interesting behaviour associated with their reduced dimensionality. Because the behaviour of materials at nanoscale abruptly changes, researchers are exploiting this property for developing applications in medicines, electronics, biotechnology, chemistry, etc. The preparation of submicro and nanostructures with defined characteristics or functional properties is a fast growing area of material science.

Nanostructured materials with controlled architectures are desirable for many applications in optics, electronics, biology, medicine, and energy/chemical conversions [8]. Highaspect-ratio metal nanostructures, nanorods, nanowires, and nanotubes are the subject of much

DOI: $10.5121 /$ ijoe.2012.1203 
current study. Nanoscale opens up new ways to study their physical and electronic properties. Nanowires exhibit a wide range of unique properties, including tunable band gaps, ballistic transport, optical anisotropy, and strong excitonic effects. There are potential applications in which a metal or metal-containing composite nanowire can replace a more conventional device, for example, in microelectronic circuits or magnetic memories. More interestingly, as quasi-onedimensional (1-D) nanostructures, metal nanowires and nanotubes reveal new physical and chemical phenomena that are a direct consequence of their size, shape, and reduced dimensionality. These novel properties promise ultimately to be of even greater technological impact because they will enable genuinely new kinds of devices and applications. It is therefore not surprising that for the past 20 years nanowires have emerged as one of the most active fields of research in material science. Nanowires can be fabricated by various techniques that include lithographic patterning $[9,10]$, vapour transport techniques [11, 12], template based-synthesis method [13, 14] and other synthesis methods $[15,16]$. The template based synthesis involving electrodeposition is more promising owing to its specific advantages of low cost and control over the nanowire properties via changing the electrolyte composition, $\mathrm{pH}$, temperature, and applied potential/current [17]. High aspect ratio nanowires synthesized by electro-deposition into templates have revealed very interesting behaviours associated with their reduced dimensionality. There has been a huge interest in the study of magnetic nanowires, in which the characteristic dimensions are comparable to the scaling lengths in magnetism or spin polarized transport [18].

Electrodeposition technique offers the advantage of allowing not only the fabrication of nanowires made from single metal but also complex structures, such as multi layers. Indeed, nanowires consisting of altering magnetic (cobalt) and nonmagnetic layers (copper) have been prepared and investigated [19-20]. Electrodeposition of several metals and semiconductors has been performed using alumina and polymeric track etched membrane templates. Electrodeposition is normally preceded by coating one side of the membrane with a noble metal [21-30].The optical properties of nanowires have been studied extensively by employing different optical characterization and analytical techniques. Metallic nanowires exhibit interesting plasmon absorption effect. Research has shown that the energy of the surface plasmon band is sensitive to various factors such as particle size, shape, composition, surrounding media and inter-particle interactions [31].

In this study, a simple and low cost electrodeposition method for synthesis of $\mathrm{Ni}$ nanowires using AAO membrane on copper substrate has been used. Also, for comparative study, $\mathrm{Ni}$ thin film is synthesized using the same electrodeposition method. The optical properties of Ni nanowires and thin film have been investigated by using a Helium-Neon ( He-Ne) laser. For capturing the optical images of the samples, a high resolution camera is used.

\section{METHODOLOGY}

Investigation is divided into two phases; fabrication and characterization of $\mathrm{Ni}$ nanowires and $\mathrm{Ni}$ thin film. Investigations of optical properties of $\mathrm{Ni}$ nanowires and $\mathrm{Ni}$ thin film deposited on copper substrate is observed by using reflected He-Ne laser light. Ni nanowires and thin film were produced by electrodeposition method. A simple two electrode system is used for electrodeposition process shown in Fig.1. 


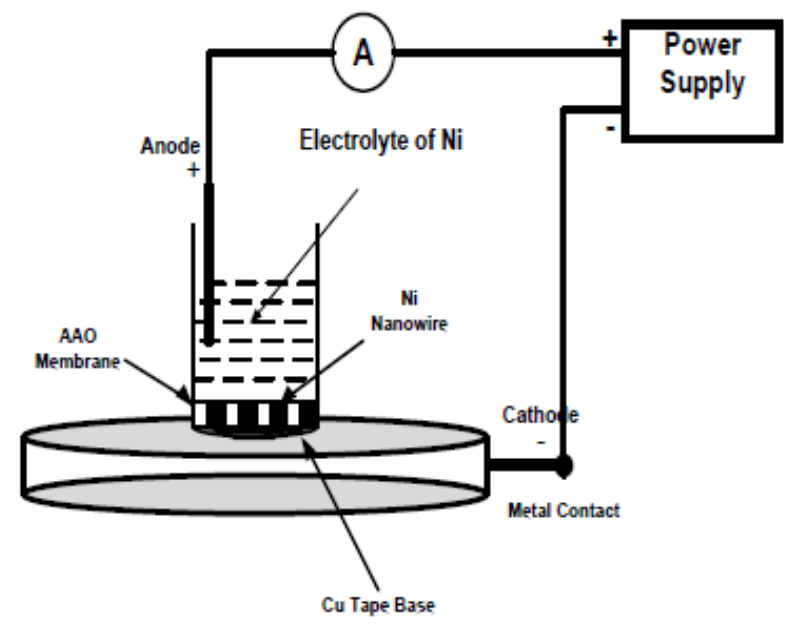

Figure.1. Schematic of two electrode cell for deposition.

Electrolyte for the deposition of nanowires comprised of nickel sulphate, nickel chloride and boric acid. The electrolyte is prepared in $20 \mathrm{ml}$ distilled water containing $0.2 \mathrm{M}$ of $\mathrm{NiSO}_{4} \cdot 6 \mathrm{H}_{2} \mathrm{O}$, $0.2 \mathrm{M}$ of $\mathrm{NiCl}_{2} \cdot 6 \mathrm{H}_{2} \mathrm{O}$, and $0.1 \mathrm{M}$ of $\mathrm{H}_{3} \mathrm{BO}_{3}$. Commercially available AAO membranes with pore size of $100 \mathrm{~nm}$ were used to for the formation of Ni nanowires. Pt electrode is used as anode and copper substrate acted as cathode for the deposition process. Copper tape is used as a substrate to deposit Ni ions. Membrane is placed on the substrate in such a way that there exist no air bubble between the membrane and copper substrate. Electrolyte is poured in the system and voltage of $2 \mathrm{~V}$ is applied. Change in current density during the deposition is carefully monitored. Current density variation during deposition time of Ni nanowires is shown Fig.2.

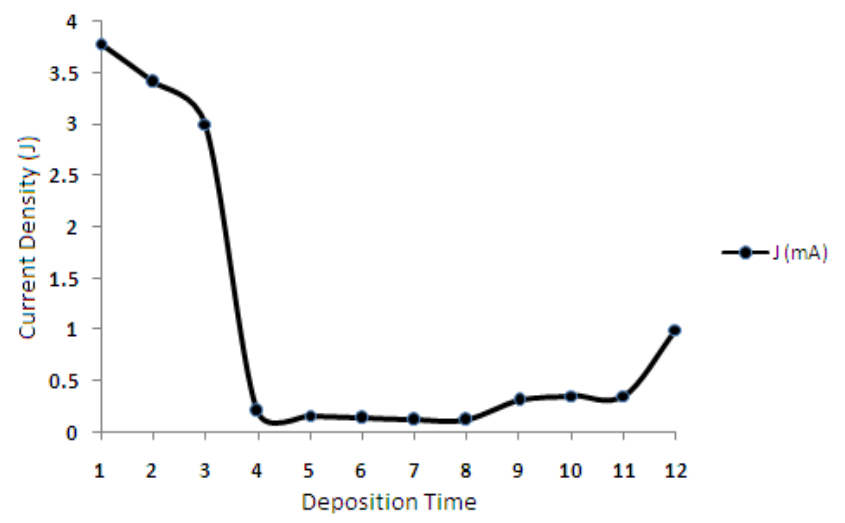

Figure 2. Current density (J) variation over deposition time.

When electrodepositions process is over, the AAO membrane is dissolved by using sodium hydroxide $(\mathrm{NaOH})$. The deposition process is again carried out to synthesize Ni thin film using same setup without AAO membrane. Figure 3 shows the morphology of the substrate after deposition of nickel nanowire and thin film on it. It is evident from change in the morphology of copper substrate that material deposition is occurred on it. 


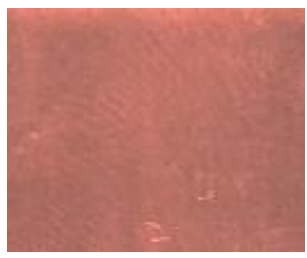

(a)

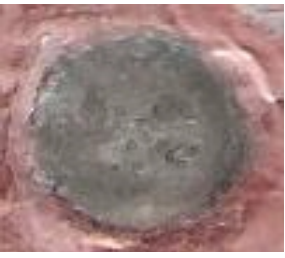

(b)

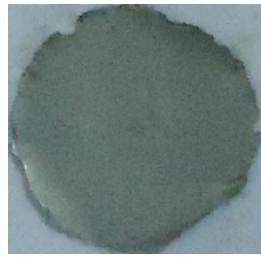

(c)

Figure 3. (a) Morphology of $\mathrm{Cu}$ substrate before deposition, (b) with Ni nanomaterial,

(c) Ni thin film.

When depositions of the $\mathrm{Ni}$ nanowires were confirmed from the morphology of the sample, the samples were further analyzed with other characterization techniques. SEM of the samples is obtained. Fig. 4 shows the side view of the SEM of Ni nanowires. One of the samples was chosen for further investigations.

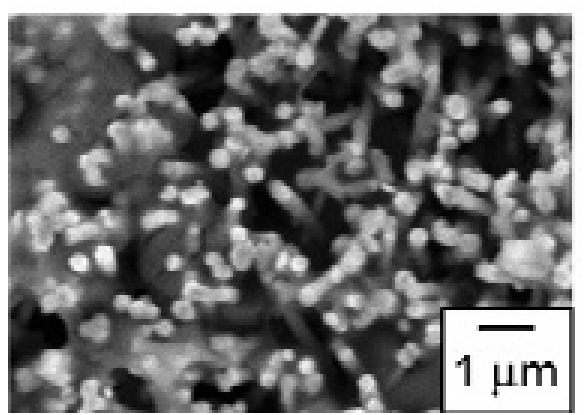

Figure 4. SEM of Nickel nanowires fabricate using electrodeposition

In the next part of the experiment $\mathrm{He}-\mathrm{Ne}$ laser is directed on the copper substrate containing $\mathrm{Ni}$ nanowires. Laser light is focused on the portion of substrate where nanomaterial is present. The laser light source is made stationary and images of the reflection of the He-Ne laser is taken from high resolution camera at different angles.

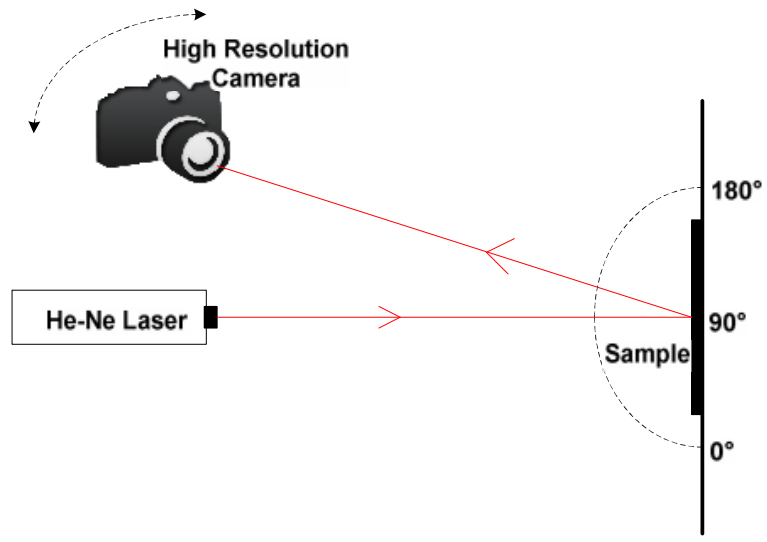

Figure 5. Schematic of the setup for analysing reflective property of Ni nanowires and Ni thin film deposited on $\mathrm{Cu}$ substrate. 
The schematic of the experiment is shown in Fig. 5. The whole experiment is done in dark room to avoid interferences from other optical sources. Angle ranging from $10^{\circ}$ to $170^{\circ}$ is marked on the horizontal surface. A rotating stand with the high resolution camera is mounted on it, so that reflected laser light from the copper substrate containing Ni nanowires can be captured. The same set of experiment is conducted using Ni thin film.

\section{RESULTS AND DISCUSSION}

SEM confirms the synthesis of vertical Ni nanowires. Since the nature of Ni material is magnetic, it is expected that nanowire will attract each other, which is evident from the SEM. He-Ne laser is directed on the portion of the copper substrate to investigate the optical properties of nickel nanowires, where nanostructured material is deposited.
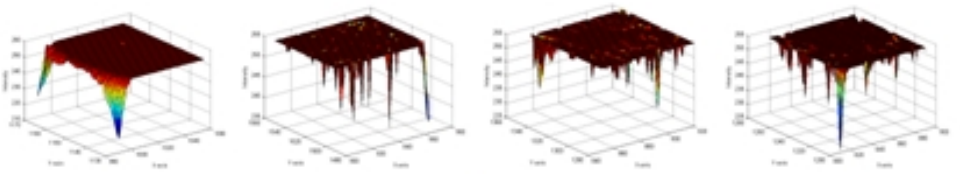

(i)
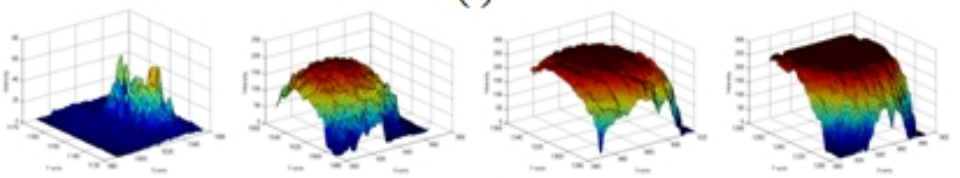

(ii)
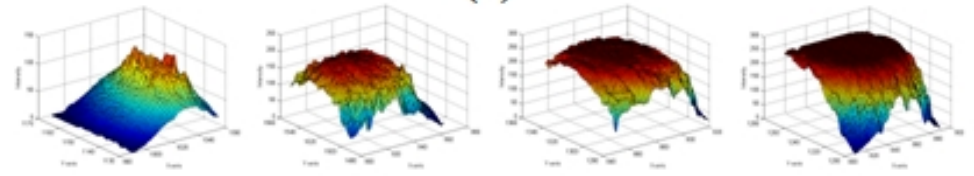

(iii)
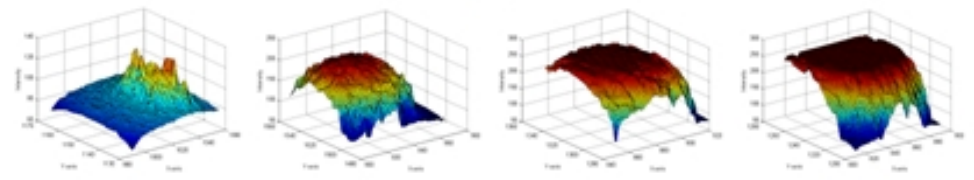

(iv)

Figure 6. 3D graph of intensity of red (i), green (ii), blue (iii), and gray (iv) components over 30x30 pixel size substrate containing Ni nanowires.

He-Ne source is kept stationary at a distance $30 \mathrm{~cm}$ from the substrate mounted on the straight platform. Images taken at different angle are processed using image processing technique. The images were cut into $30 \times 30$ pixels size. These images were further processed for red, green, and blue colour components intensity. The colour images are desaturated in order to nullify the effect of surrounding optical sources. The intensities of images for red, green, blue, and gray scale components of the processed images over $30 \times 30$ pixel size for $30^{\circ}, 60^{\circ}, 90^{\circ}, 120^{\circ}$, are shown in Fig. 6. The plots in the first row show intensity pattern for red component. It may be observed from these plots that the intensity over the selected area remains almost constant except few sharp valleys. The second row shows the blue component; where heaps of intensity at different points is clearly visible. Similar patterns may be observed in the other rows. The average intensity of desaturated images of Ni nanowires at different angles is shown in Fig. 7. The same set of experiment is carried out for Ni thin film and average intensity is calculated as shown in Fig. 8. 
From these results it is seen that behaviour of $\mathrm{Ni}$ nanowires is smooth, regular, and more convergent, but in case of $\mathrm{Ni}$ thin film more irregularities and maxima is observed at different angles.

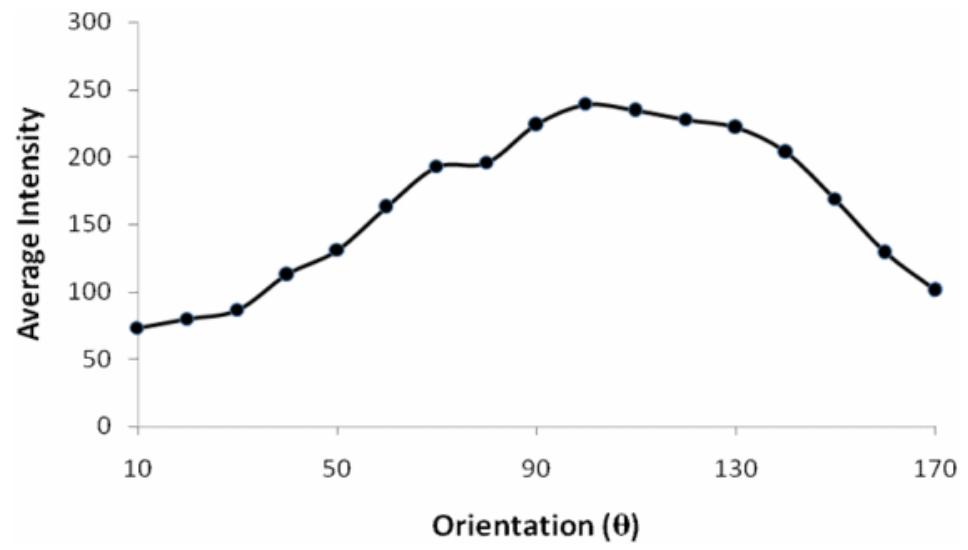

Figure 7. Average intensity of desaturated image over 30 × 30 pixel size of $\mathrm{Cu}$ substrate containing $\mathrm{Ni}$ nanowires at different angles.

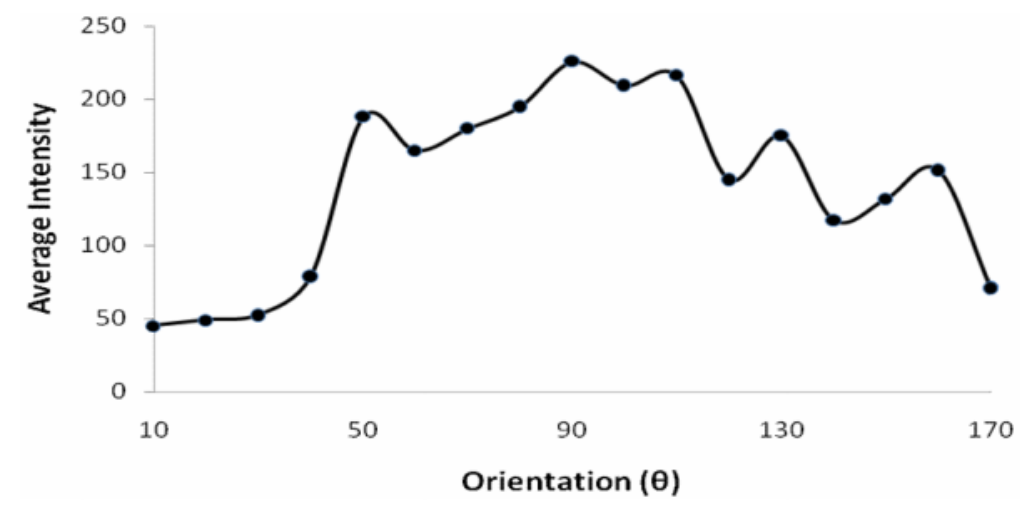

Figure 8. Average intensity of desaturated image over $30 \times 30$ pixel size of Ni thin film at different angles.

\section{CONClusion}

Electrodeposited Ni nanowires were successfully synthesized by template assisted method. The effect of direction of incident of $633 \mathrm{~nm}$ wavelength He-Ne laser light for investigating the optical properties of Ni nanowires and thin film was studied. The analysis of the results showed that the reflection pattern of the nanowires and thin film is different. Optical characterization of Ni nanowires showed a smooth pattern on both side of the normal to the sample. On the other hand, the pattern observed for the Ni thin film was not so smooth and contained a number of peaks and valleys. This interesting behaviour of Ni nanowires may be further explored for developing optical sensing systems. 
International Journal on Organic Electronics (IJOE) Vol.1, No.2, July 2012

\section{REFERENCES}

[1] C.L. Peterson, (2004) "Nanotechnology: from Feynman to the grand challenge of molecular manufacturing," Technology and Society Magazine, IEEE, Vol. 23, pp. 9-15.

[2] E. Regis, (1995) Nano: The Emerging Science of Nanotechnology, Little Brown.

[3] M. Krummenacker, J. Lewis, (1995) Prospects in Nanotechnology: Toward Molecular Manufacturing, Wiley.

[4] B. Nelson, L. Dong, A. Subramanian, D. Bell, (2007) "Hybrid Nanorobotic Approaches to NEMS, Springer Tracts in Advanced Robotics," Springer, Berlin/Heidelberg, pp. 13-174.

[5] N. Nandhagopal, A. A. Simpson, J. R. Gurnon, X. Yan, T. S. Baker, M.V. Graves, J. L. Van Etten, M. G. Rossmann, (2002) "The structure and evolution of the major capsid protein of a large, lipid containing DNA virus,” Proc. Natl. Acad. Sci. ,Vol. 99 , pp.14758-14763.

[6] G.E. Fryxell, (2007) "Self-Assembly, Productive Nanosystems a Technology Roadmap," Foresight Institute, pp. 51-52.

[7] D. Rebolj, M. Fischer, D. Endy, T. Moore, A. Sorgo, (2011) "Can we grow buildings? Concepts and requirements for automated nano- to meter-scale building," Advanced Engineering Informatics, Vol. 25, pp.390-398.

[8] T. L. Sounart, J. Liu, J. A. Voigt, J. W. P. Hsu, E. D. Spoerke, Z. Tian, Y. B. Jiang, (2006) "Sequential Nucleation and Growth of Complex Nanostructured Films," Advanced Functional Materials, Vol. 16, pp. 335-344 .

[9] P R Bandaru and P Pichanusakorn, (2010) "An outline of the synthesis and properties of silicon nanowires," Semiconductor science and technology, Vol. 25.

[10] S. F. Abd. Rahman, U. Hashim, M. N. Md. Nor, A. M. Mohamed Nuri, M. E. A. Shohini, and S. Salleh, (2009) "Nanowire Formation Using Electron Beam Lithography," Proc. AIP Conf., Vol. 1136, pp.504-508.

[11] G. Malandrino, S. T. Finocchiaro, R. L. Nigro, C. Bongiorno, C. Spinella, and I. L. Fragala, (2004) "Free-Standing Copper(II) Oxide Nanotube Arrays through an MOCVD Template Process," Chem. Mater., Vol.16, pp.5559.

[12] S. Y. Bae, H. W. Seo, and J. H. Park, (2004) "Vertically Aligned Sulfur-Doped ZnO Nanowires Synthesized via Chemical Vapor Deposition," J. Phys. Chem. B, vol.108, pp.5206.

[13] C. R. Martin, (1996) "Membrane-Based Synthesis of Nanomaterials," Chem. Mater., Vol. 8, pp.1739-1746.

[14] T. R. Kline, M. Tian, J. Wang, A. Sen, M. W. H. Chan, and T. E. Mallouk, (2006) "TemplateGrown Metal Nanowires," Inorganic Chemistry, Vol. 45, pp.7555-7565.

[15] C. Xu, G. Xu, Y. Liu, and G. Wang, (2002) "A simple and novel route for the preparation of ZnO nanorods," Solid State Communication, Vol.122, pp.175-179.

[16] D. Zheng, S. Sun, W. Fan, H. Yu, C. Fan, G. Cao, Z. Yin, and X. Song, (2005) "One-Step Preparation of Single-Crystalline $\beta-\mathrm{MnO} 2$ Nanotubes," J. Phys. Chem. B, Vol. 109, pp.1643916443.

[17] S. K. Chakarvarti, (2006) "Science and art of synthesis and crafting of nano/microstructures and devices using ion-crafted templates: a review," Proc. SPIE.

[18] A. Fert and L. Piraux, (1999) "Magnetic nanowires," J. Magn. Magn. Mater, Vol. 200, pp.338.

[19] L. Piraux, J. M. George, J. F. Despres, C. Leroy, E. Ferain, R. Legras, K. Ounadjela, and A. Fert, (1994) "Giant magnetoresistance in magnetic multilayered nanowires," Applied Physics Letters, Vol. 65, pp.2484.

[20] François de Menten de Horne, L. Piraux, and S. Michotte, (2005) "Fabrication and physical properties of $\mathrm{Pb} / \mathrm{Cu}$ multilayered superconducting nanowires," Applied Physics Letters, Vol.86.

[21] L. V. Gomez, S. Cattarin, P. Guerriero, M. Musiani, (2007) "Preparation and electrochemical characterization of $\mathrm{Ni}+\mathrm{RuO} 2$ composite cathodes of large effective area," Elsevier, Electrochimica Acta, Vol. 52, pp.8055.

[22] M.J. Zheng, L.D. Zhang, G.H. Li, W.Z. Shen, (2002) "Fabrication and optical properties of largescale uniform zinc oxide nanowire arrays by one-step electrochemical deposition technique,"Elsevier, Chemical Physics Letters, Vol. 363, pp.123.

[23] R. Inguanta , S. Piazza, C. Sunseri, (2009) "Synthesis of self-standing Pd nanowires via galvanic displacement deposition," Elsevier, Electrochemistry Communications, Vol.11, pp.1385. 
International Journal on Organic Electronics (IJOE) Vol.1, No.2, July 2012

[24] H. Pan, B. Liu, J. Yi, C. Poh, S. Lim, J. Ding, Y. Feng, C.H.A. Huan, J. Lin, (2005) "Growth of Single-Crystalline $\mathrm{Ni}$ and Co Nanowires via Electrochemical Deposition and Their Magnetic Properties," J. Phys. Chem. B, Vol.109, pp. 3094.

[25] M. E. ToimilMolares, V. Buschmann, D. Dobrev, R. Neumann, R. Scholz, I. U. Schuchert, J. Vetter, (2001) "Single-Crystalline Copper Nanowires Produced by Electrochemical Deposition in Polymeric Ion Track Membranes," Advanced Materials, Vol.13, pp. 62.

[26] M.Tian, J. Wang, J. Kurtz, T. E. Mallouk, and M. H. W. Chan, (2003) "Electrochemical Growth of Single-Crystal Metal Nanowires via a Two-Dimensional Nucleation and Growth Mechanism," Nano Letters, Vol.3, pp. 919-923.

[27] Y. Konishi, M. Motoyama, H. Matsushima, Y. Fukunaka , R. Ishii, Y. Ito, (2003) "Electrodeposition of $\mathrm{Cu}$ nanowire arrays with a template," Elsevier, J. of Electroanalytical Chemistry, Vol.559, pp.149.

[28] D.Grujicic, B.Pesic, (2002) "Electrodeposition of copper: the nucleation mechanisms," Pergamon, Electrochimica Acta, Vol. 47, pp. 2901-2912.

[29] Parveen Lehana, Saleem Khan, and Sandeep Arya, “ Fabrication And Investigations Of Cufe Nanowires Based Sensors," International Journal of VLSI and Signal Processing Applications, Vol. 1, Issue 2 , pp. 32-37, 2011.

[30] Parveen Lehana, Manpreet Kaur, Ashok Kumar, Sandeep Arya, Saleem Khan, "Effect of $\lambda$ in the em visible region on the reflective properties of nickel nanowires," International Journal on Organic Electronics (IJOE), Vol.1, No.1, pp. 13-19, 2012.

[31] J. Sarkar, G. G. Khan, A Basumallick, (2007) "Nanowires: properties, applications and synthesis via porous anodic aluminium oxide template,” IAS, Bull. Mater. Sci., Vol. 30, pp. 271-290. 